\title{
IMPROVED THROUGHPUT WITH COOPERATING FUTURISTIC AIRSPACE MANAGEMENT COMPONENTS
}

\author{
Patricia Glaab, NASA Langley Research Center, Hampton, VA
}

\begin{abstract}
An experiment was conducted to integrate airspace management tools that would typically be confined to either the en route or the terminal airspace to explore the potential benefits of their communication to improve arrival capacity. A NASwide simulation was configured with a new concept component that used the information to reconfigure the terminal airspace to the capacity benefit of the airport. Reconfiguration included a dynamically expanding and contracting TRACON area and a varying number of active arrival runways, both automatically selected to accommodate predicted volume of traffic. ATL and DFW were selected for the study.

Results showed significant throughput increase for scenarios that are considered to be over-capacity for current day airport configurations. During periods of sustained demand for ATL 2018, throughput increased by 26 operations per hour $(30 \%)$ and average delay was reduced from 18 minutes to 8 minutes per flight when using the dynamic TRACON. Similar results were obtained for DFW with 2018 traffic levels and for ATL with 2006 traffic levels, but with lower benefits due to lower demand.
\end{abstract}

\section{Introduction}

In current day operations in the National Airspace (NAS), the area in the vicinity of the airport that uses radar surveillance to manage aircraft during the arrival and departure flight phases is designated as the Terminal Radar Approach Control (TRACON) airspace. Outside these TRACONs, Center controllers manage en route aircraft. Control for arriving flights transitions from the authority of the Center to that of the TRACON at the arrival fix, or from TRACON to Center at the departure fix for departing flights. Within the TRACON, arriving aircraft travel from the arrival fix to the runway along standard terminal arrival routes (STARs). For departures, the paths are called Standard Instrument Departures (SIDs). The arrival and departure routes are fixed paths through the airspace and are designed to keep arrivals separated from departures as long as aircraft adhere to them. TRACON controllers estimate distances between sequential aircraft traveling the same route with the assistance of monitoring tools, and they issue speed adjustments or vectoring instructions to pilots to maintain required wake spacing between aircraft when necessary. These operations are handled efficiently through training and practice, and rely on repetition for safety. Static sub-regions called Sectors are defined to identify the exact area of responsibility for each controller. As the aircraft travels through its departure, en route, and arrival phases, it passes through many Sectors assisted by many controllers.

In future NAS visions, this job of managing the trajectories of aircraft is facilitated with computer automation. Computer automation potentially offers the safety of current day NAS operations without relying on rigidity of the airspace boundaries. When the algorithms in use are intelligent and robust, the computer does not need repetition and statically defined airspace regions to achieve safe operation. With this in mind, the interaction and communication of airspace management tools can be re-engineered. Traditionally, en route tools were designed to assist Center controllers, and arrival/departure tools were designed to assist TRACON controllers with limited communication between the systems. In a more futuristic NAS, however, information sharing between these tools and more fluid regions of control authority may lead to significant efficiency gains.

In preparation for this futuristic NAS vision, research in NASA Langley's Aeronautics and Systems Analysis Branch (ASAB) is investigating the potential benefits of integrated en route and terminal airspace management tools. The simulation that was created to conduct the experiment leveraged prior results which quantified the arrival capacity benefits possible by expanding the area of authority for trajectory adjustment by the TRACON. In the prior experiment, significant throughput benefit was achieved with a larger, static TRACON radius [1]. For this experiment, a dynamic TRACON airspace was enabled to expand when more capacity was needed to accommodate arrivals and to contract when 
low arrival volume allowed to minimize the duration of imposed Required Time of Arrival (RTA) deadlines on flights. The dynamic nature of such a changing boundary would be challenging for human controllers. For this reason, the concept relies on automation technologies to enable the operation.

\section{Background}

In the configured simulation, the system that replaces the role of the human TRACON controllers is called the Merging and Spacing (M\&S) component. Just as with human TRACON controllers, the authority of the M\&S system is confined to the terminal airspace. $M \& S$ contains several sub-components, one of which is an arrival scheduler. The arrival scheduler's job is to handle each arrival flight that crosses the TRACON boundary by trial planning its path along the STAR route in consideration of all previously scheduled traffic. The arrival scheduler inspects the crossing time at each crossing fix and tests whether the aircraft will be far enough from the aircraft in front (and in back if it was being merged into a gap in traffic) to be in compliance with FAA regulated wake spacing. If not, it continues trial planning alternate options until it finds a trajectory that is both compliant with spacing requirements and is also within the performance limits of the aircraft being managed. These crossing times are stored as new reservations to the arrival fixes (for reference for subsequently scheduled fights) and the crossing times are issued to the aircraft as RTAs.

In a prior experiment, the radial distance from the arrival airport at which the arrival scheduler component began its planning (the "planning radius") was varied between simulation runs to quantify the impact of this radius on throughput. The test radii ranged from 50 nautical miles (nmi) to $200 \mathrm{nmi}$. In the expanded radius runs, the larger distance between scheduling and landing increased flexibility to arrange and adjust trajectories within the performance limits of arriving aircraft. This increased flexibility translated to significant throughput benefit when coupled with the intelligent arrival scheduler, and the throughput improvement was achieved without reduced wake spacing and without adding runways (Figures 1 and 2).

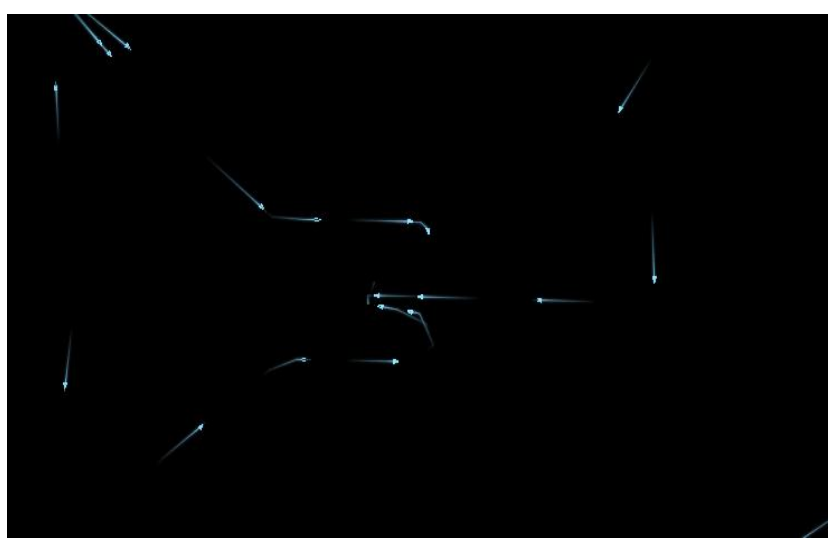

Figure 1. Peak Efficiency for the $50 \mathrm{nmi}$ Planning Radius

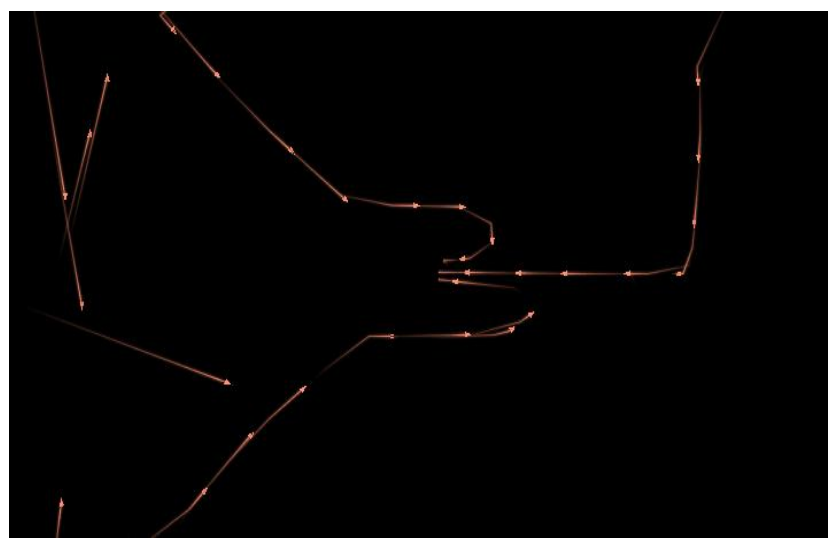

Figure 2. Peak Efficiency for the $130 \mathrm{nmi}$ Planning Radius

The benefit increased until the radius reached $130 \mathrm{nmi}$. At that point, the arrival stream was nearly as densely populated as the imposed wake spacing allows, and no additional benefit was achieved by expanding the radius beyond $130 \mathrm{nmi}$.

However, the expanded planning radius also meant that scheduled aircraft were locked into RTAs earlier, potentially at the cost of more efficient trajectory choices. When arrival volume was high, this was a reasonable tradeoff. However, when volume was low, it placed a trajectory efficiency burden on arrivals without adding value since the demand could have been satisfied with a smaller planning radius. The concept system for this experiment was developed to address this problem by using a dynamic airport and airspace configuration. The static radius of control for the arrival scheduler was replaced with an expandable radius. Within a given run, this dynamic radius only expanded when necessary to accommodate the imminently arriving traffic and contracted to the current day size of approximately 50 nautical miles (nmi) when lower 
volume allowed. The runway configurations were simultaneously tailored to support the varying radius.

Though the concept targets arrival flights only, one of the benefit cases is improved departure throughput (when the user selects a configuration option to minimize the number of arrival runways in use). In this case, opportunistic expansion of the planning radius allows the same volume of traffic to be landed with fewer runways. This leaves more departure runway time available for airports that delegate runways exclusively for either arrivals or departures (like runway 28 in Atlanta).

The dynamic nature of such a changing boundary would be challenging for human controllers who rely on static boundaries and clear delineations of controller airspace for safety. However, it does offer future potential for the National Airspace System (NAS) as it transitions from fully humancontrolled to a system with more autonomous flight operations. In a future visionary NAS with nearly full autonomy, this type of system could serve as an advanced arrival planning system with a tailored airspace management area to meet the changing volume in the course of a day. In a nearer term future that has many unequipped aircraft, the dynamic arrival planning radius could serve as a demarcation line. Before crossing this line, autonomous aircraft must either commit to a set of arrival reservations or accept transfer of authority for trajectory management to the human controller. It would be, effectively, a "choose no later than" threshold. The pilot of the autonomous aircraft would have the greatest flexibility to select an efficient trajectory when volume was low and the dynamic radius was small. As volume and the corresponding planning radius increased, the pilot's ability to remain autonomous would require commitment to a set of reservations earlier in the flight with the knowledge that trajectory adjustments might have to be made (at the cost of performance) to meet the RTA deadlines. If the autonomous aircraft crossed the planning horizon boundary without committing to a reservation set, autonomy would be forfeited and authority would revert to the human controller.

Some of the required functionality for such a system is possible through enhancements to existing tools. Human controllers employ Traffic Flow Management (TFM) tools and procedures that monitor flight schedules and airport conditions to ensure that arrivals do not exceed the capacity of the airports to which they are destined and apply ground holds or in-flight delays as needed [2]. In the notional system, TFM would maintain its current role with additional knowledge of the location of the dynamic arrival scheduling radius which would be the extent of its in-flight delay authority. Traffic Management Advisor (TMA) is already available to coordinate aircraft arrival schedules, but is currently used to provide insight to human controllers. In the notional system, TMA would be expanded to allow autonomous aircraft access to available arrival reservations while still outside the planning horizon boundary. Unlike the current TMA, the autonomous aircraft would optionally be allowed to select Scheduled Times of Arrival (STAs) earlier than their initial Estimated Times of Arrival (ETAs) at their discretion. TMA would continue its current role to provide insight to human controllers as they managed flights without autonomous reservations and simply monitored flights with autonomous reservations.

Figure 3 shows the cooperative airspace management components that were integrated to create the simulation experiment. The critical new enabling technology for this vision is a system that determines the size of the dynamic arrival planning radius. For this experiment, that system is called the Terminal Airspace Configuration System, or TACS. 


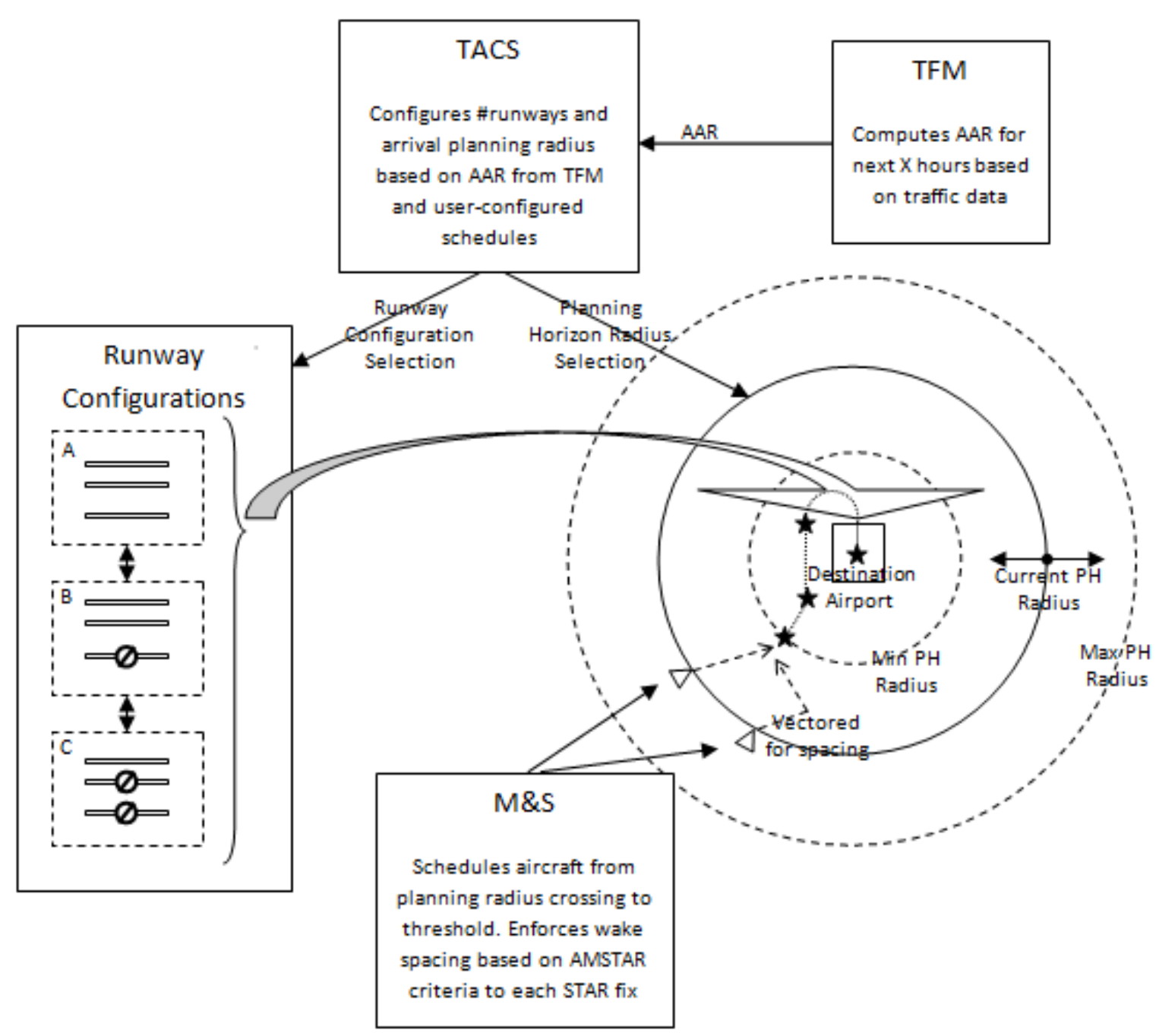

Figure 3. Cooperative Components in a Futuristic NAS

\section{Terminal Airspace Configuration System (TACS)}

The TACS component is responsible for computing the best planning radius and runway configuration to meet the predicted volume of arrival traffic. The best planning radius is the smallest size required to meet the predicted volume. The best runway configuration uses the fewest number of runways required in conjunction with that radius. A capacity-constrained TRACON might want to minimize the number of arrival runways to leave more runways available for departures or to reduce the personnel requirement for managing the extra runways. 


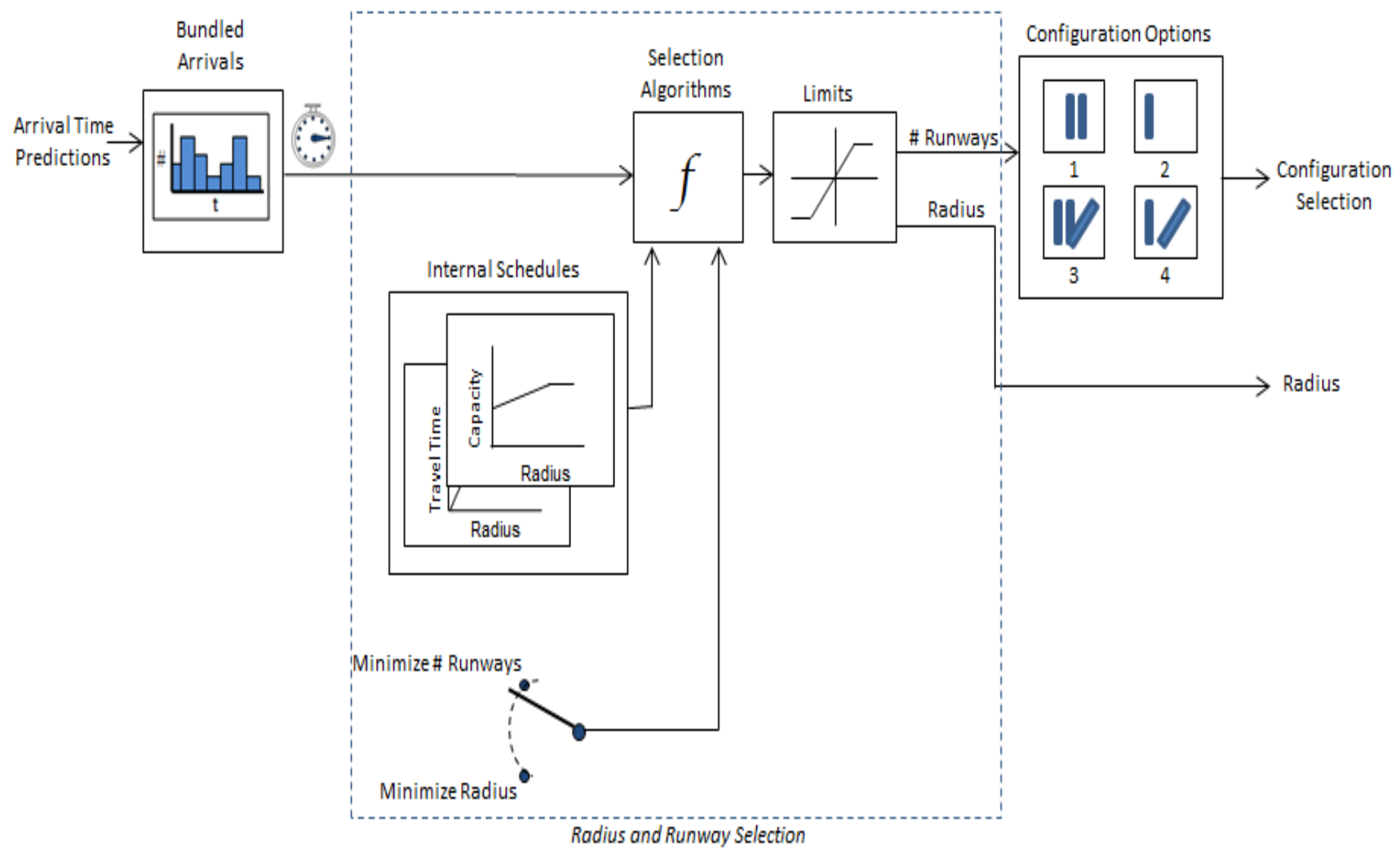

Figure 4. High-Level Control Diagram of TACS

Figure 4 presents a high-level control diagram for TACS. The block labeled "Bundled Arrivals" identifies the gathering and processing of predicted flight schedules. TACS receives specific arrival time information for flights within the look-ahead window from TFM. For this simulation, groups of arrival traffic are bundled into time blocks (or "bins") for realism since predicted arrival demand information is presented to controllers in time blocks in current realworld ATC operations. TMA's Load Graph Window is an example of this type of tool, and updates in 10 minute intervals. The duration of individual traffic bins is a configurable parameter and additionally determines the size of the TACS update interval. This is required to insure that any window can result in a configuration change if needed. At the beginning of each TACS update cycle, the bundled arrivals are computed and passed to the computational algorithms, represented by the block labeled "Radius and Runway Selection".

A two-step process is used to determine the radius and runway selection. First, the individual bins are analyzed to determine the configuration suitable for each specific time window. Then, results for all individual bins are inspected to determine which future window must be acted upon immediately to adequately accommodate all traffic within the lookahead period. This allows the radius to expand in advance of the traffic, and larger radii are triggered farther in advance than smaller radii.

The number of runways requested by TACS is limited to the maximum number available in the configuration file. A limit is also applied to the rate at which the radius can contract to avoid overtaking aircraft that are already planned. Expansion has no rate limit.

The last TACS step matches the number of runways requested to an available airport configuration (the "Configuration Options" block in Figure 3) and informs the arrival scheduler of the new radius and runway configuration. This completes the TACS processing. Until the next TACS update frame, the Arrival Scheduler uses the most recent radius as a reference for where to assume control of the arrival trajectory (Figures 5 and 6). 


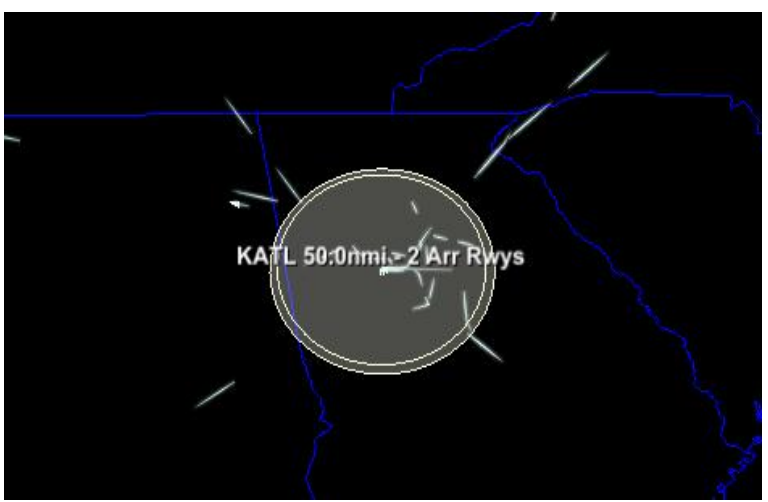

Figure $5.50 \mathrm{nmi}$ radius and 2 runways

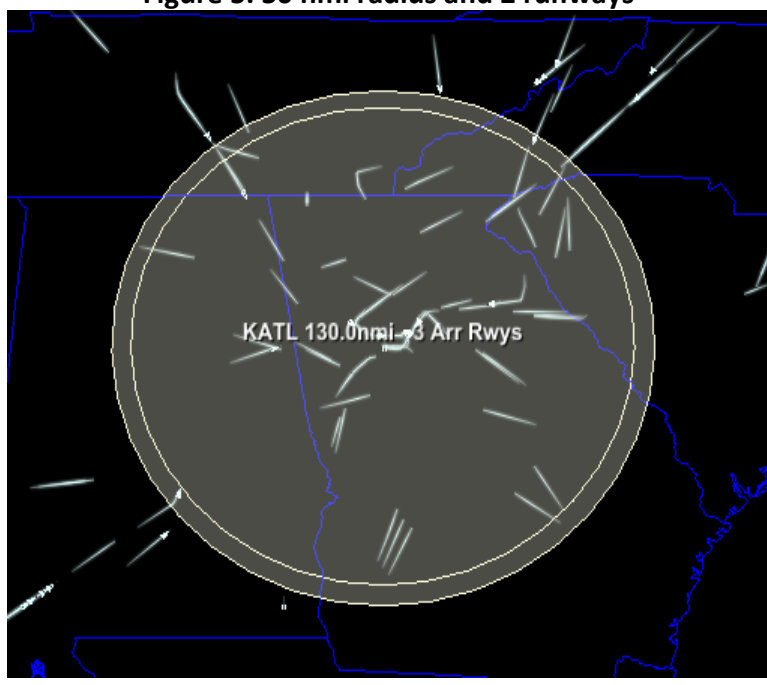

Figure $6.130 \mathrm{nmi}$ radius and 3 runways

\section{Configuration Options}

In some cases, the same volume can be handled with either an increase in the number of runways OR with an increase in the planning radius. For these intervals, the system needs to know which to minimize. The TACS component allows either objective to be set as an option in a configuration file. In practice, a capacity-constrained Terminal Radar Approach Control (TRACON) might want to minimize the number of arrival runways to leave more runways available for departures or to reduce the personnel requirement for managing the extra runways. Conversely, the priority might be placed on reducing the footprint of the arrival airspace to allow autonomous aircraft to commit to arrival reservations later in their flight to reduce uncertainty that might impact their fuel efficiency (for example, due to imperfect wind predictions).

Figures 7 and 8 present the different planning radii and runways that result for arrival volumes ranging from 0 to 130 aircraft per hour for the 2 options.

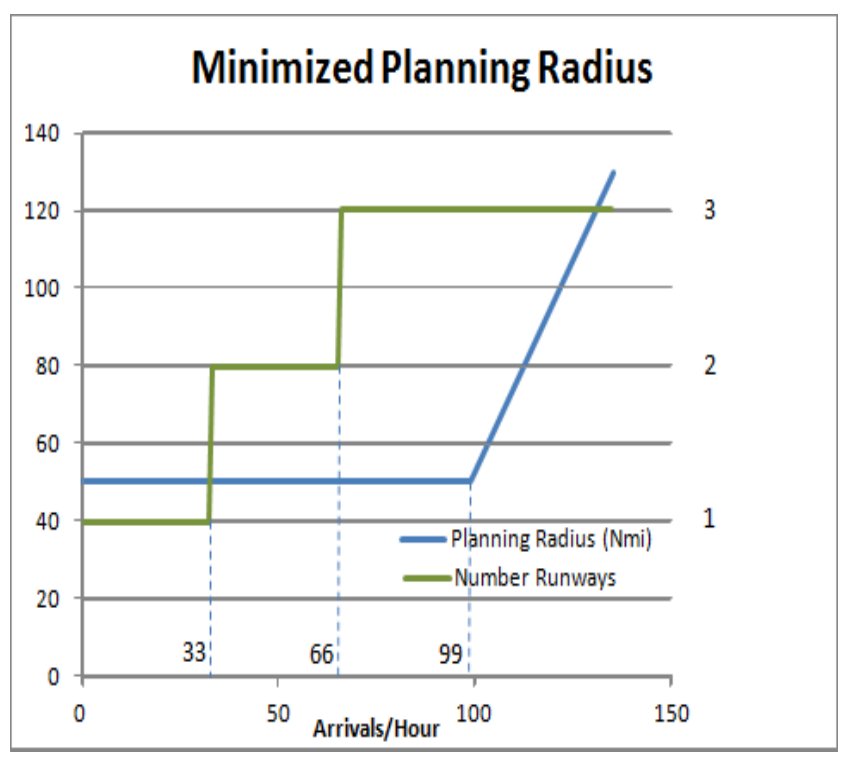

Figure 7. Minimized Radius Schedule

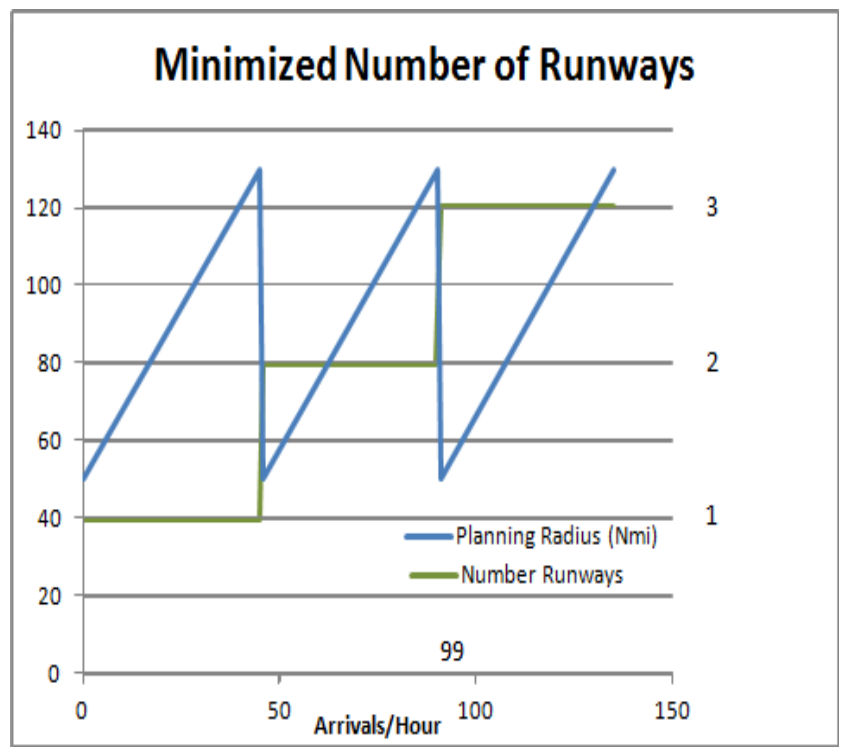

Figure 8. Minimized Runways Schedule

\section{Lead Time Calculation}

TACS estimates the time-to-go for flights at any radius from the airport. This time-to-go is used as a lead time to initiate a radius expansion in advance of traffic that will need to schedule at that expanded radius. If this lead time is too short and the expansion occurs too late, the arrival scheduler will not have the distance and flexibility it needs to schedule those flights without incurring extra delay. Conversely, if 
the expansion occurs too early, the radius will be larger than needed and the surrounding airspace will be unnecessarily burdened. Ideally, the radius expands just before the targeted flights cross it.

Faster moving aircraft require more lead time for radius change than slower aircraft. Also, there is inherent uncertainty in the estimated times of arrival (ETAs) from TFM because the ETAs are based on the shortest arrival procedures, which the flights often do not get during high volume when longer downwind legs are used for spacing. The arrival planning radius is symmetric around the airport. However, a flight arriving to the planning radius from the leeward end of the runway has a different time to touchdown than a flight arriving from the windward end (Figure 9). These uncertainties and variations were accommodated within the TACS algorithm's lead time calculation.

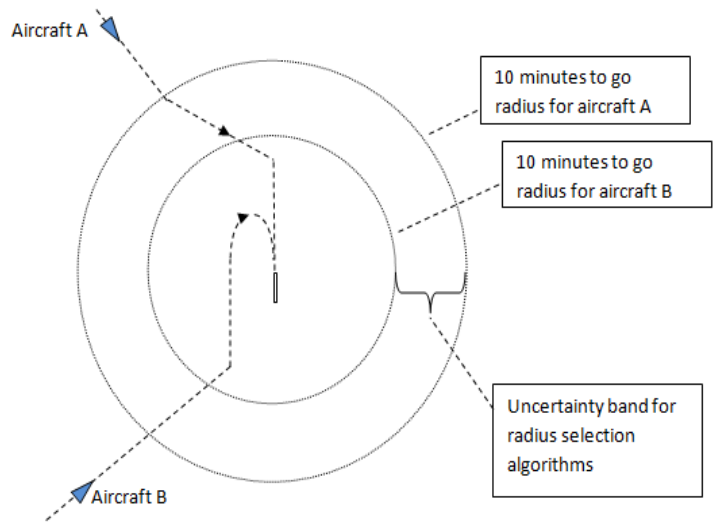

Figure 9. Ten Minutes-to-Go for Different Routes provided a generalized schedule of time-to-go for a given path distance-to-go (Figure 10). The path distance-to-go refers to the path traversed across the STAR fixes.

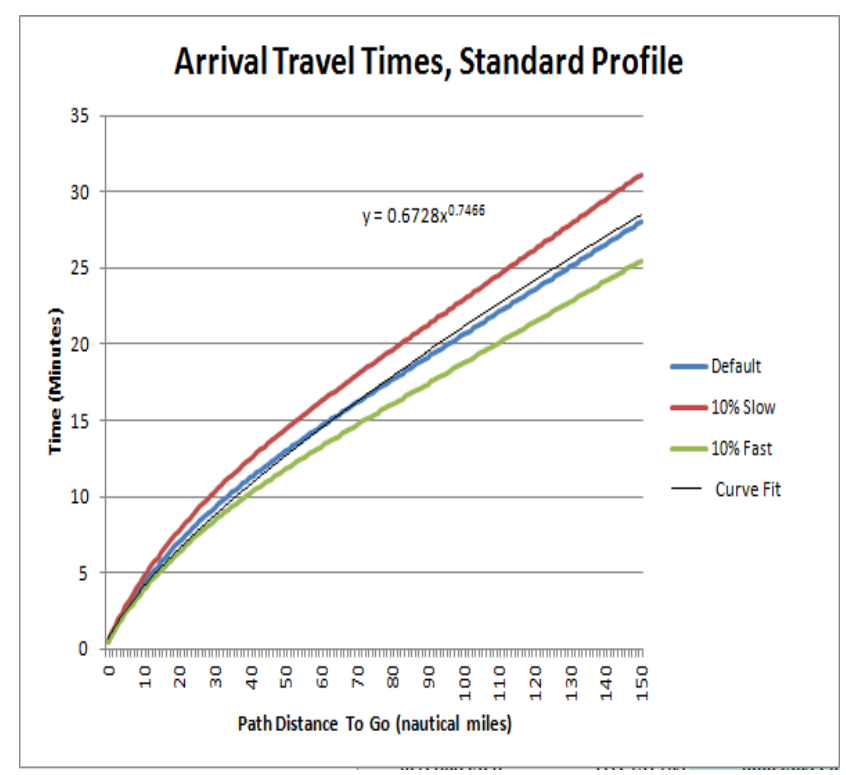

Figure 10. Standard Profile Arrival Times

The path distance-to-go was correlated to a radial distance-to-go. To do this, STAR route measurements for DFW from Reference [1] were used (Table 1). Because of the variation in STAR route lengths, a single ideal lead time is not feasible. For this experiment, the time was increased to accommodate the worst case (the leeward side arrivals).

The estimated lead time for TACS is based on parameter identification from prior research [1] which

\begin{tabular}{|c|c|c|c|c|}
\hline Route Description & Measurement Waypoint & $\begin{array}{c}\text { Radial Distance } \\
\text { (nmi) }\end{array}$ & $\begin{array}{c}\text { Path Distance } \\
\text { (nmi) }\end{array}$ & $\begin{array}{c}\text { Difference } \\
\text { (Correction) } \\
\text { (nmi) }\end{array}$ \\
\hline Cedar Creek, 17C, 5 mile & HOWDY & 43.41 & 55.84 & 12.43 \\
\hline Cedar Creek, 17C, 10 mile & HOWDY & 43.41 & 64.86 & 21.45 \\
\hline Glen Rose, 18L, 5 mile & FEVER & 45.93 & 58.8 & 12.87 \\
\hline Glen Rose, 18L, 10 mile & FEVER & 45.93 & 67.97 & 22.04 \\
\hline Bowie, 18L, 10 mile & UKW & 54.85 & 58.04 & 3.19 \\
\hline Bowie, 18L, 20 mile & UKW & 54.85 & 64.48 & 9.63 \\
\hline Bonham, 17C, 10 mile & KARLA & 44.04 & 47.41 & 3.37 \\
\hline Bonham, 17C, 20 mile & KARLA & 44.04 & 53.92 & 9.88 \\
\hline
\end{tabular}

Table 1. DFW Arrival Route Lengths 
The final algorithms worked equally well for the Atlanta traffic, and so both airports use the same set of equation for their lead time calculation.

\section{The Arrival Scheduler}

The arrival scheduler is an efficient first-comefirst-served (FCFS) scheduler that can either expedite or delay flights within performance limitations specified by Base of Aircraft Data (BADA)[6]. Additionally, the scheduler can either issue following instructions for aircraft that can be assigned a leader with a compatible trajectory or can assign required time of arrival (RTA) deadlines for crossing fixes when no suitable leader is available. For aircraft that have not yet crossed the first STAR waypoint, the scheduler imposes a path stretch maneuver when necessary to add delay for spacing to that first STAR fix. For this simulation, the scheduler initiates its planning for an arriving flight as soon as the flight crosses the planning radius.

\section{Configuration Update Interval Impact on the Computed Radius}

The selection for the configuration update interval $(5,10$, or 15 minutes for this experiment) directly impacts the number of possible radius values. Since the traffic is separated into time bins that match the update interval, the bin size shrinks in direct proportion to the update interval. An indirect result of this is that the number of possible expansion radius options decreases with the shrinking bin size.

This dependency is best illustrated with an extreme example where the duration of each bin is reduced to the point where it is shorter than the time required between sequentially landing aircraft. In this extreme case, each bin is limited to a corresponding radius option of either the minimum (50 for this experiment) or the maximum (130 for this experiment). This is because an empty bin corresponds to "all capacity available" which drives the radius calculation to the least restrictive (the minimum) value. However, the addition of a single aircraft corresponds to "all capacity used" which drives the radius to the most restrictive (the maximum) value. As the bin duration increases, the number of radius options increases as demonstrated by the radius values observed in simulation testing (Table 2).

\begin{tabular}{|l|l|}
\hline $\begin{array}{l}\text { Update Period } \\
\text { (Minutes) }\end{array}$ & Expanding Radius Options (nmi) \\
\hline 1 & 50,130 \\
\hline 5 & $50,70,97,110,123,130$ \\
\hline 10 & $50,70,83,90,97,110,123,130$ \\
\hline 15 & $50,52,57,61,70,79,88,97,106,123,130$ \\
\hline
\end{tabular}

\section{Simulation Description}

\section{ACES Host Simulation Version}

The Airspace Concepts Evaluation System (ACES) [3] NAS-wide simulation was used to test the concept system. The version used was the January 2012 delivery of ACES 7.1 with Merging and Spacing (ACES with M\&S) which can simulate a full day of traffic from runway to runway. The M\&S plugin was developed by Intelligent Automation, Inc. (IAI) and contains both an intelligent arrival and departure scheduling tool and an interval and merge management component to enforce spacing regulations for aircraft on the same arrival route within the planning radius [4]. The M\&S component was based on Airborne Merging and Spacing for Terminal Arrivals (AMSTAR) research [5] by Barmore, Abbot, and Kristnamurthy. ACES uses physical trajectories modeled by the Kinematic Trajectory Generator (KTG), also from IAI, which references BADA performance capabilities. The TACS component software was added to this version of ACES.

\section{Study Airports and Traffic Sets}

Four traffic sets were constructed by isolating arrival traffic destined for the target airports from each the 2006 and 2018 Baseline Day traffic files [7]. The 2006 sets approximates current day traffic volume and the 2018 sets provides approximately 1.3 times the current day traffic (or " $1.3 \mathrm{X}$ ") for each of the two test airports, Atlanta's Hartsfield Jackson (ATL) and Dallas Fort Worth (DFW). DFW and ATL are similar in that they both use a "four corner post" arrival configuration where aircraft approaching the airports cross fixes at the northeast, northwest, southeast, and southwest corners of the Terminal Radar Control (TRACON) 
area. DFW is a non-capacity-constrained airport, and does not become constrained even with predicted 2020 traffic volume (according to the FAA's FACT 2 report [8] on Capacity Constraints in the NAS). ATL is listed as an airport predicted to be over-capacity before 2020. FACT 2 also notes that the addition of the third runway in Atlanta solved the capacity-constraint situation that existed prior to its installation, indicating that current day Atlanta requires periodic 3-runway operation to avoid being over-capacity. Use of these four scenarios (DFW 2006, DFW 2018, ATL 2006, and ATL 2018) allowed the testing of comparable airspaces under a range of four traffic loading conditions ranging from under to over-capacity.

Both traffic sets were collected on a clear, high volume day in the NAS consistent with Visual Flight Rule (VFR) conditions for the two study airports. However, the simulation aircraft were constrained to Instrument Flight Rule (IFR) following standards all the way through runway touchdown. This intentionally stresses the arrival scheduler. Atlanta, for example, advertises an acceptance rate of 103 aircraft per hour to the surrounding Center during IFR conditions and 126 aircraft per hour during VFR conditions. This is because during VFR conditions, pilots can be cleared for visual approach which allows them to close on lead aircraft on final approach at their discretion.

\section{Simulation Delay versus Real-World Delay}

It is important to note that simulation delay metric are useful for run-to-run comparisons, but do not correspond to delay as reported in real-world air transport operations because the systems tally delay differently. The simulation considers a flight to be "delayed" in any case where the simulated time of flight exceeds the best time of flight possible. The best time of flight possible occurs when the aircraft is offered the shortest possible arrival routing option (no delays for merges or following, no extensions beyond the shortest possible final) and departs exactly at the preplanned time. This reporting criterion usually causes the simulation delay to exceed its real-world counterpart. For example, the simulation does not allow delayed departure minutes to be recaptured en route. The simulation also does not provide schedule padding to compensate for a 10 mile final rather than a 5 mile final. This could be added to marry the realworld with the simulation cases, but it would make the analysis more difficult without adding value. So the delay reporting should be treated as a means of assessing runs against each other, only.

\section{Airborne Delay and Diverted Flights}

When the M\&S arrival scheduler delays a flight for spacing before the first STAR fix, it applies a path stretch maneuver. The path stretch is increased in 10 second increments as needed, and this metric is tallied for the runs as "Airborne Delay". In real-world operations, a holding pattern is more typical when large delays are required, but the delay granularity possible with a path stretch is much finer than for a holding pattern and so was preferred for this simulation. When the path stretch delay became unrealistically large, M\&S diverted the aircraft to another airport. Diverted flights occurred when the system experienced prolonged periods of over-capacity and acted as an effective "pressure release valve" for excess flights. The airborne delay for the diverted flights was removed from the reported totals.

The delay results presented are normalized to the delay per flight. For this metric, the highest volume period of the day was isolated based on the throughput time histories for each traffic set, the period between the $12^{\text {th }}$ and $24^{\text {th }}$ simulation hour, which corresponded to approximately 8 am to 8 $\mathrm{pm}$. For the high volume period, the total measured delay was divided by the number of landed flights to compute the delay per flight.

\section{Experiment Matrix}

Table 3 summarizes the variety of runs executed.

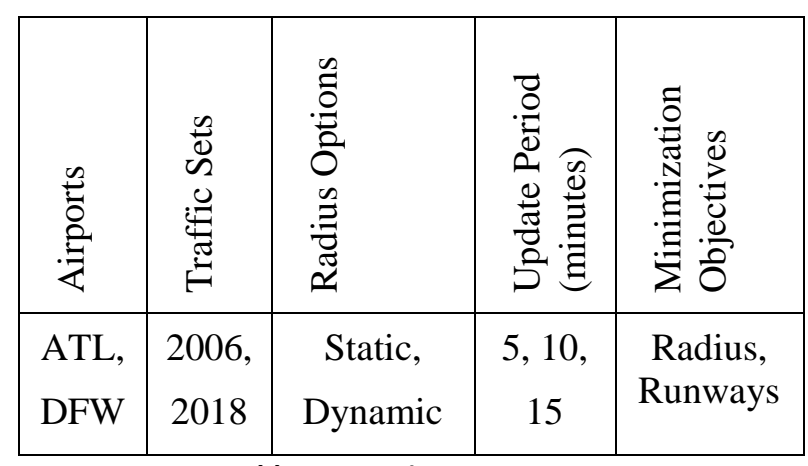

Table 3. Experiment Run Targets 


\section{Results}

\section{Impact of Update Interval}

The ATL 2018 traffic set was used to assess the impact of the update interval on the performance of the system since it created the longest period of sustained demand due to the overcapacity volume of traffic. For this test, the dynamic radius was configured with the update period of TACS $(5,10$, or 15 minutes) as the sole difference between runs. Both the time history results (Figure 11) and the computed peak and mean throughput results (Table 4) showed no significant difference between the two methods. Since more frequent updates limit the number of radius options (see prior discussion in Section "Configuration Update Interval Impact on the Computed Radius"), the 15-minute interval was used for static to dynamic radius comparison testing.

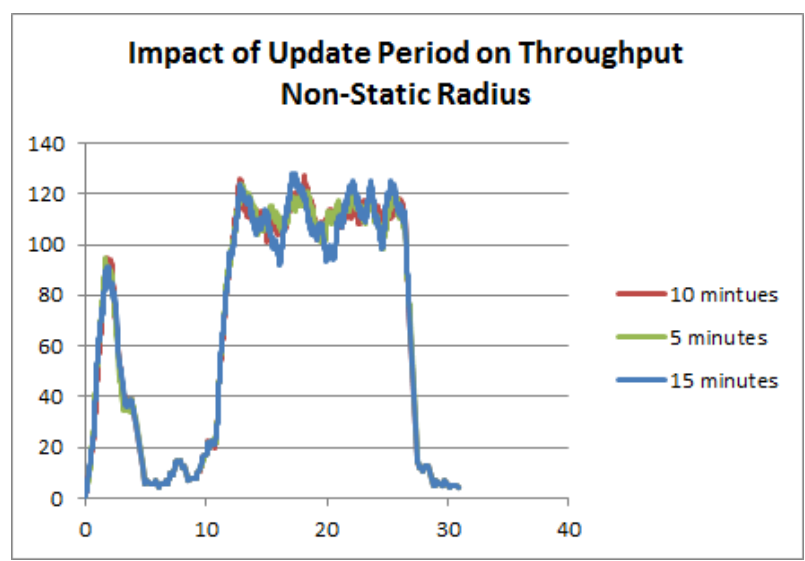

Figure 11. Impact of Update Period

\begin{tabular}{|c|c|c|c|c|}
\hline 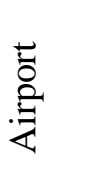 & 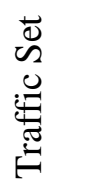 & 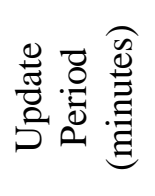 & 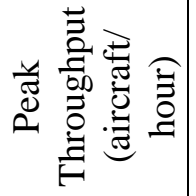 & 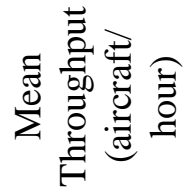 \\
\hline ATL & 2018 & 15 & 128 & 110.9006 \\
\hline ATL & 2018 & 10 & 127 & 112.0288 \\
\hline ATL & 2018 & 5 & 124 & 111.8726 \\
\hline
\end{tabular}

\section{Minimization Objective}

The system was tested to ensure that the configuration option to minimize either the number of runways or the size of the planning radius works as intended without impacting the system throughput. The result was a nearly identical throughput time history (Figure 12).

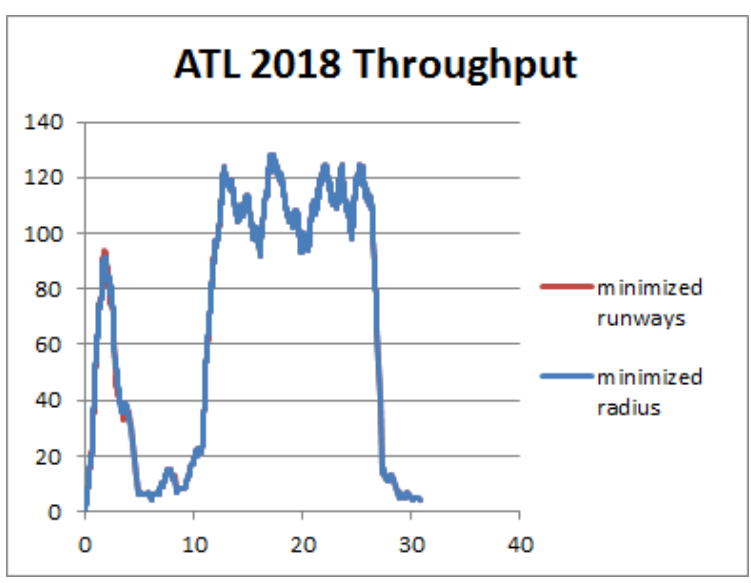

Figure 12. Throughput with Minimization Objectives

The difference between the options shows up in the time-in-configuration results as an increased percentage of time in 1-runway configuration for the minimized runway case and an increased percentage of time in a $50 \mathrm{nmi}$ radius for the minimized

case. 


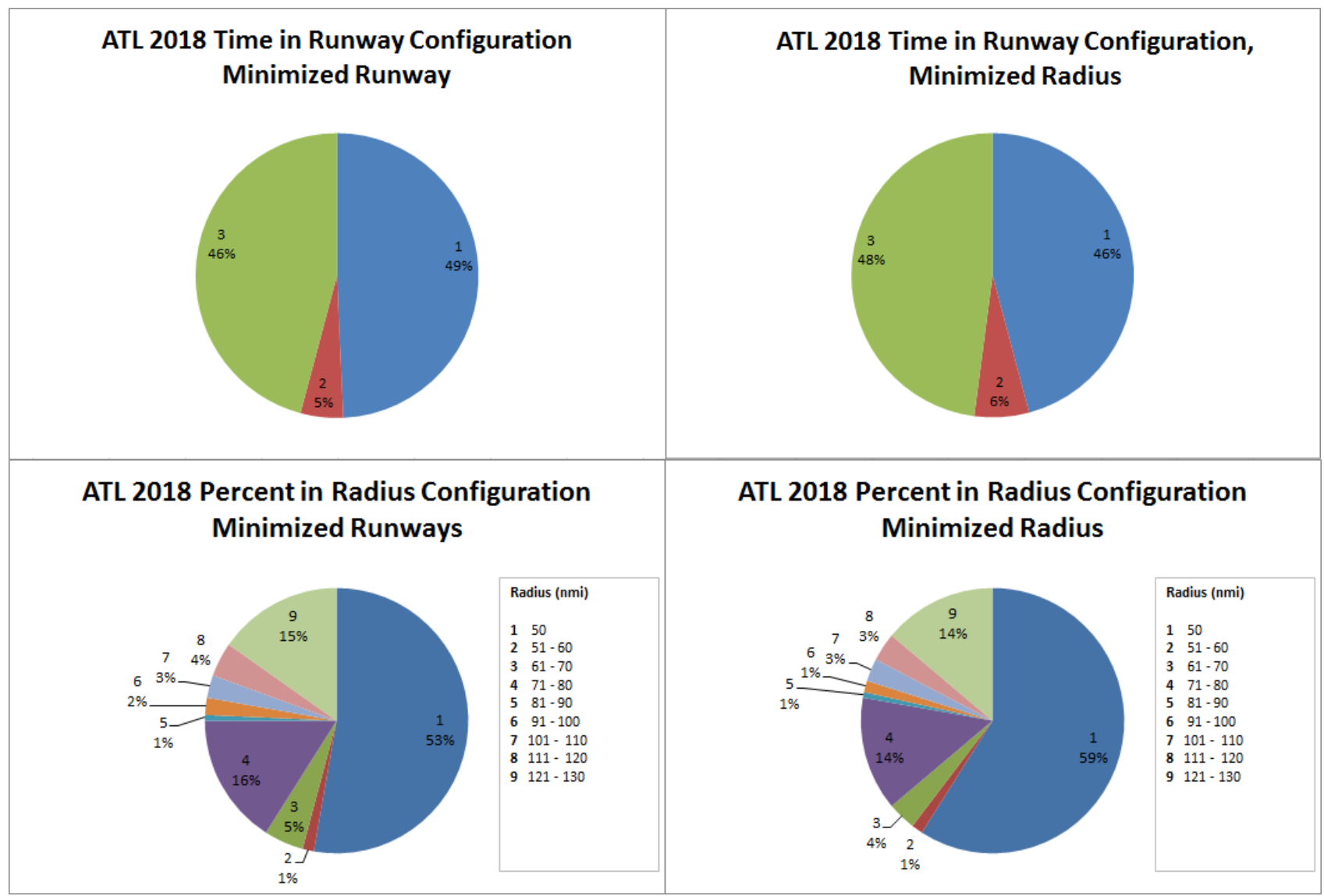

Figure 13. ATL 2018 Percentage of Time in Configuration

The time-in-configuration results are also shown for the ATL 2006 and DFW 2018 cases. Though these cases contain less traffic volume and have shorter periods of sustained demand than the ATL 2018 case, they are useful for demonstrating the configuration options possible for the system to potentially accommodate arrivals with fewer runways during high demand periods (previously discussed in Section "Configuration Options").
ATL 2006 Time in Runway Configuration Minimized Runway

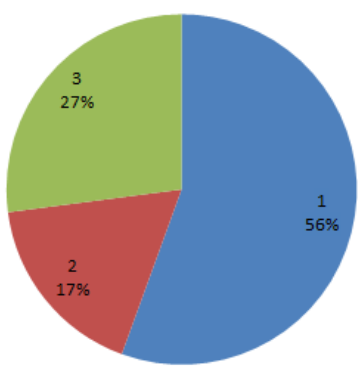

ATL 2006 Time in Runway Configuration Minimized Radius

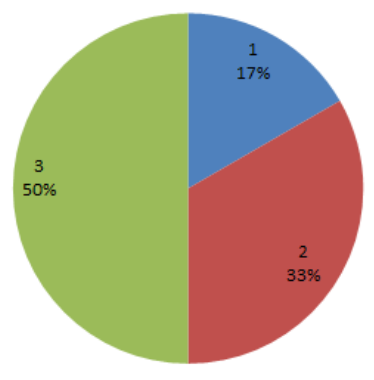




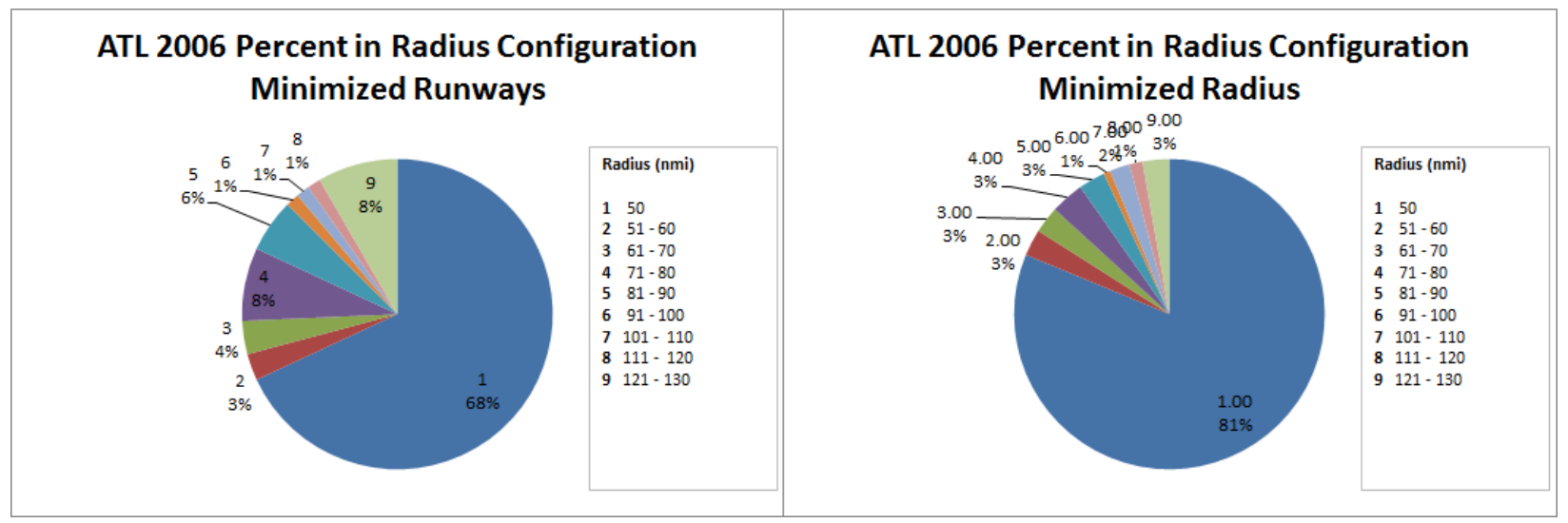

Figure 14. ATL 2006 Percentage of Time in Configuration

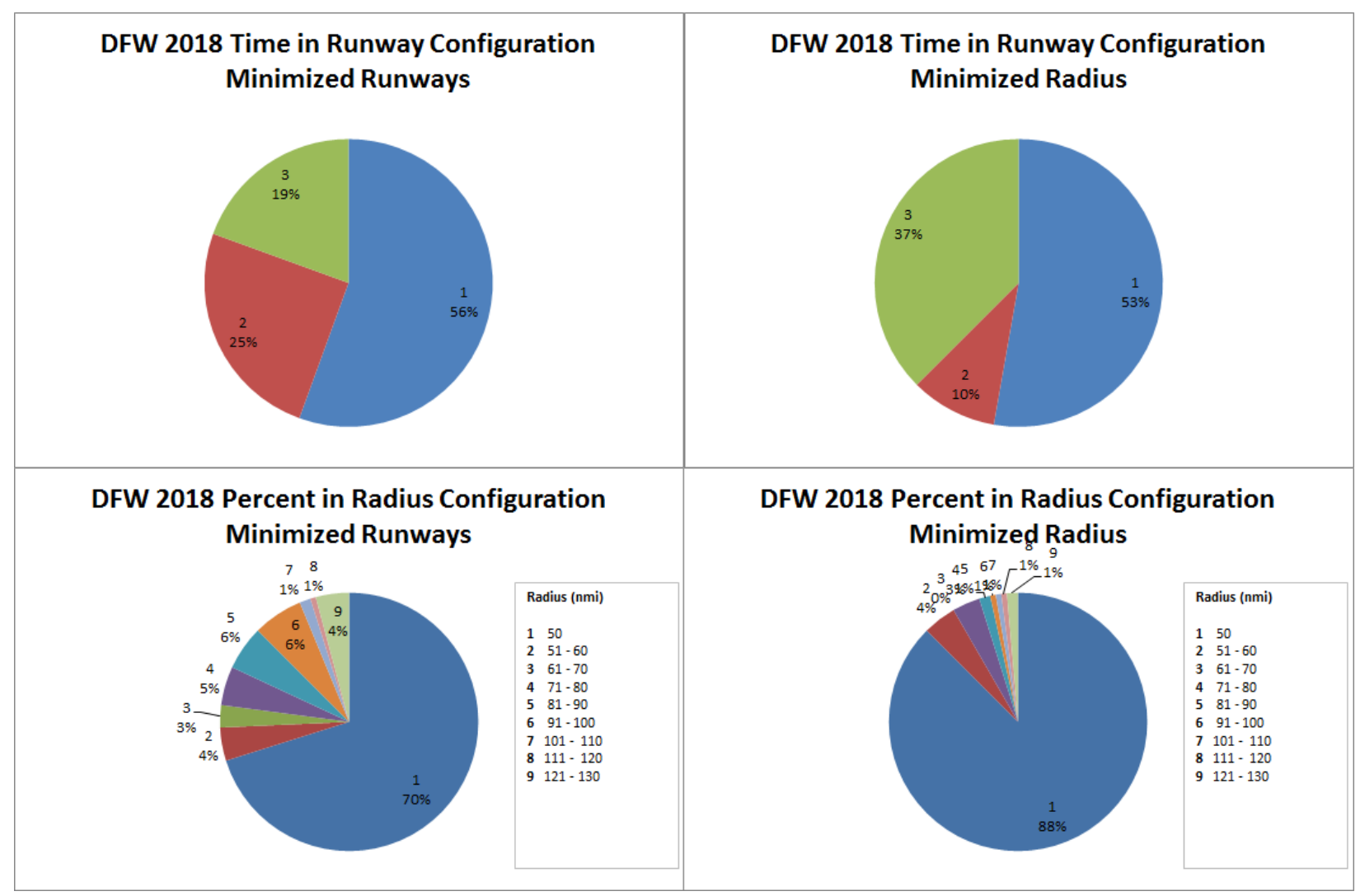

Figure 15. DFW 2018 Percentage of Time in Configuration

\section{Static Versus Dynamic Radius Tests}

The time histories for the static versus dynamic radius cases are shown in Figures $16-19$ with the associated peak and mean throughputs in Tables $5-8$. The period of sustained volume, which was used for the calculation of the mean throughput, is noted on each graph. Recall that IFR spacing restrictions are used for the simulation, though the traffic sets were captured on clear weather days when VFR conditions were in effect. The Atlanta TRACON advertises an arrival acceptance rate to the surrounding Center of 126 aircraft per hour during VFR operation and 103 aircraft per hour during IFR operation. The static versus the dynamic radius results for the highest volume case, ATL 2018, differ by an amount similar to the real world IFR versus VFR landing rates suggesting that the dynamic radius may allow VFR arrival rates under IFR spacing regulations. 


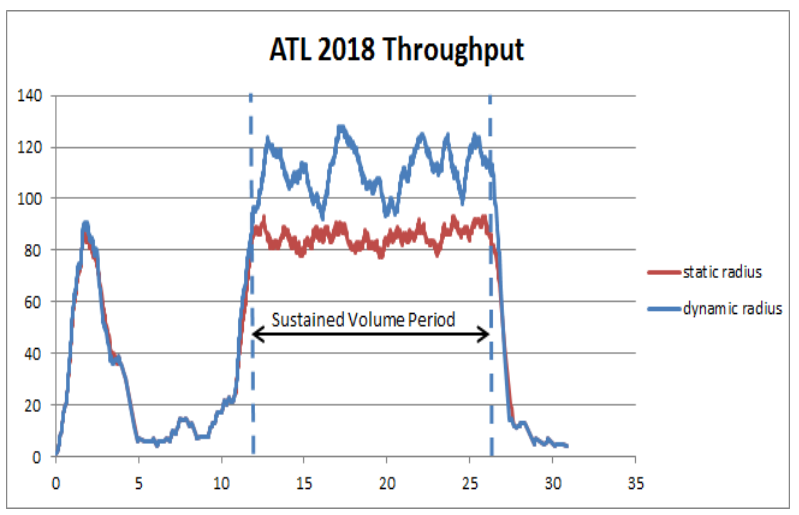

Figure 16. ATL 2018 Throughput, Static VS Dynamic

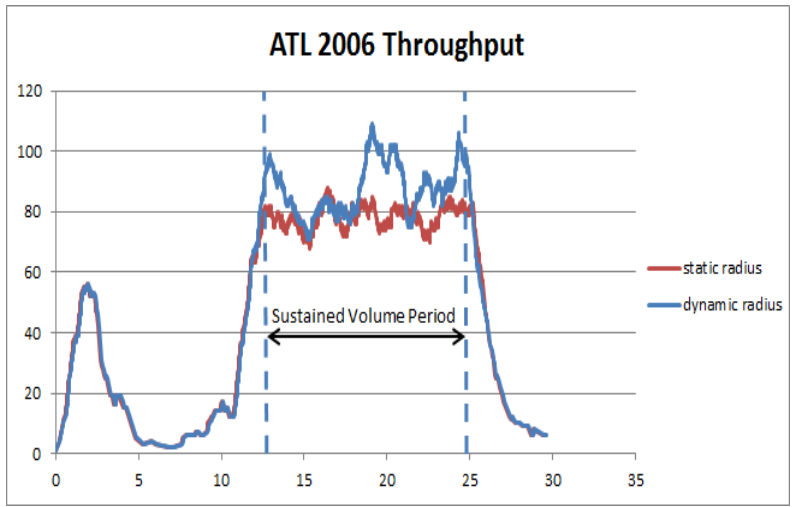

Figure 17. ATL 2006 Throughput, Static VS Dynamic

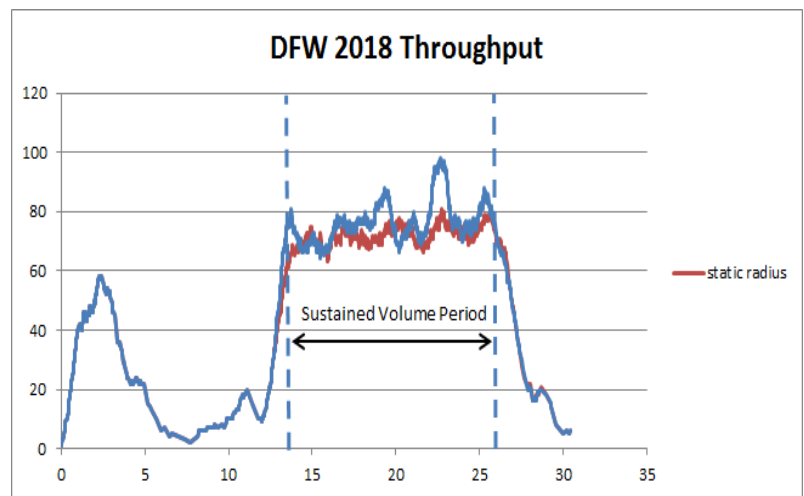

Figure 18. DFW 2018 Throughput, Static VS Dynamic

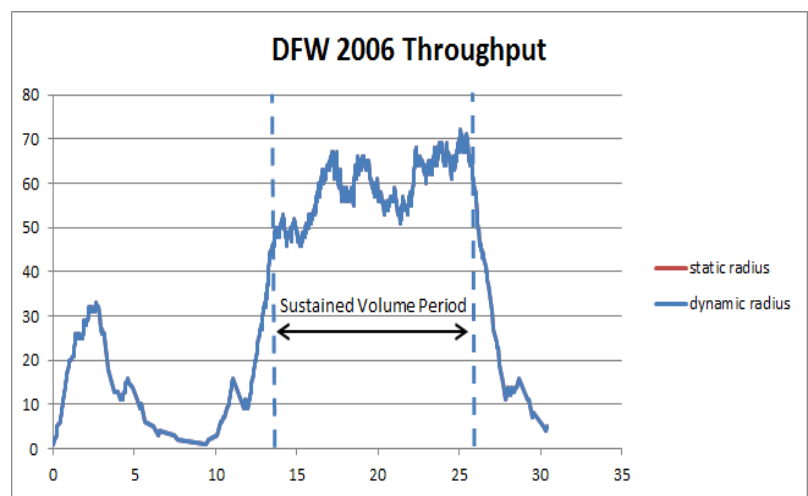

Figure 19. DFW 2006 Throughput, Static VS Dynamic

\begin{tabular}{|c|c|c|c|c|}
\hline \multicolumn{5}{|c|}{ ATL 2018 Throughput } \\
\hline 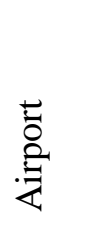 & 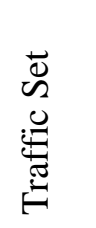 & 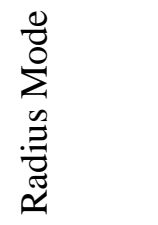 & 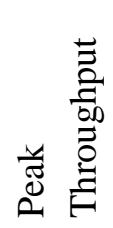 & 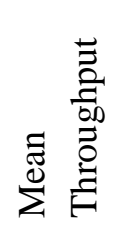 \\
\hline ATL & 2006 & Static & 93 & 84.88 \\
\hline ATL & 2006 & Dynamic & 128 & 110.90 \\
\hline
\end{tabular}

Table 5. ATL 2018 Peak and Mean Throughput

\begin{tabular}{|c|c|c|c|c|}
\hline \multicolumn{5}{|c|}{ ATL 2006 Throughput } \\
\hline 莺 & 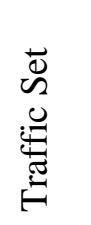 & 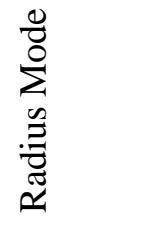 & 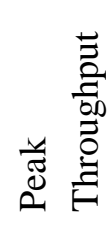 & 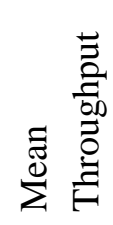 \\
\hline ATL & 2018 & Static & 88 & 78.41 \\
\hline ATL & 2018 & Dynamic & 109 & 88.06 \\
\hline
\end{tabular}

Table 6. TL 2006 Peak and Mean Throughput

\begin{tabular}{|c|c|c|c|c|}
\hline \multicolumn{5}{|c|}{ DFW 2018 Throughput } \\
\hline 䒕 & 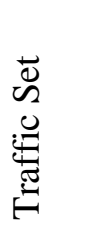 & 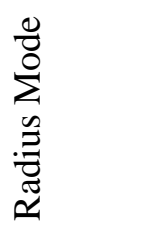 & 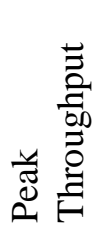 & 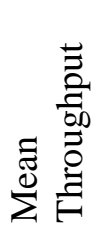 \\
\hline DFW & 2018 & Static & 81 & 72.11 \\
\hline DFW & 2018 & Dynamic & 98 & 76.93 \\
\hline
\end{tabular}

Table 7. DFW 2018 Peak and Mean Throughput

\begin{tabular}{|c|c|c|c|c|}
\hline \multicolumn{5}{|c|}{ DFW 2006 Throughput } \\
\hline 䒕 & 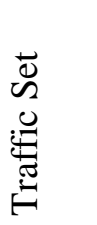 & 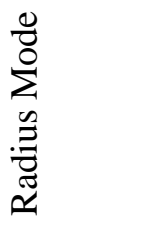 & 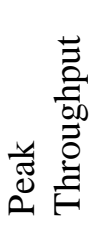 & 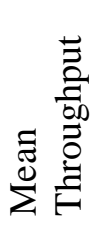 \\
\hline DFW & 2006 & static & 72 & 59.55 \\
\hline DFW & 2006 & dynamic & 72 & 59.55 \\
\hline
\end{tabular}

Table 8. DFW 2006 Peak and Mean Throughput 
The largest benefit occurs with ATL 2018 because this case has the highest traffic volume (Figure 7 and adjacent Table 5). For this overcapacity volume scenario, the mean throughput for the dynamic radius case exceeded that of the static radius case by 26 aircraft per hour (an improvement of about $30 \%$ ). Note that benefit for the other cases is only observed during periods of near-capacity or over-capacity. As the overall volume decreases, the benefit diminishes until the performance between the static and dynamic radius is identical for the DFW 2006 case. This is because the system is designed to provide capacity benefit, which cannot be realized for under-capacity traffic volume. During low volume periods (for example, DFW 2018 from 14 - 16 hours and 23 - 25 hours and during the entire DFW 2006 run), the system effectively reverts to current day operation.

For the ATL 2018 case, notice also that as the system approaches capacity (which is a lower value for the static radius than for the dynamic radius), the peaks and valleys level out. The static radius (the red trace) has very little amplitude change during the sustained volume period. This occurs when the arrival stream is nearly as fully loaded as the arrival scheduler can achieve, and additional flights must be delayed to subsequent slots which levels the traffic. The larger amplitude change between the peaks and valleys in the dynamic radius trace (in blue) demonstrate that even though this case has higher mean throughput, there is still some throughput available if more volume were supplied by the traffic set. As a general rule, the traces with greater amplitude of change indicate less delay because short term surges in volume are accommodated without having to delay flights as often.

\section{Airborne Delay}

When a flight traverses a longer STAR path than the shortest path available or when the arrival scheduler has to delay a flight using a path stretch for spacing, the time difference incurred is tallied as airborne delay. The total airborne delay during the period of sustained volume was divided by the number of landings during the same period to compute the airborne delay per flight to normalize the values relative to the different throughput values. The results for the delay per flight for the dynamic radius cases were significantly less than for the static radius cases. This means the system had improved throughput benefit concurrent with improved delay benefit. As with the throughput, the realized benefit decreased as the traffic volume decreased from the ATL 2018 to the DFW 2006 cases.

\begin{tabular}{|c|c|c|c|}
\hline Airport & $\begin{array}{c}\text { Traffic } \\
\text { Set }\end{array}$ & $\begin{array}{c}\text { Radius } \\
\text { Mode }\end{array}$ & $\begin{array}{c}\text { Delay } \\
\text { (minute/flight } \\
\text { during peak) }\end{array}$ \\
\hline ATL & 2018 & static & 18.01904 \\
\hline ATL & 2018 & varied & 8.122538 \\
\hline ATL & 2006 & static & 13.6363 \\
\hline ATL & 2006 & varied & 7.762938 \\
\hline DFW & 2018 & static & 17.39108 \\
\hline DFW & 2018 & varied & 14.38675 \\
\hline DFW & 2006 & static & 8.996451 \\
\hline DFW & 2006 & varied & 8.996451 \\
\hline \multicolumn{4}{|c|}{ Table 9. Airborne Delay } \\
\hline
\end{tabular}

\section{Diverted Flights}

Diverted flights occurred when the system experienced prolonged periods of over-capacity. This forced the arrival scheduler to apply path stretch maneuvers to delay flights. When the path stretch delay became unrealistically large for any given aircraft, the $M \& S$ component diverted the aircraft to another airport. The diverted flights contributed to neither the delay nor the throughput metrics. However, they are useful as a general indicator of the system's ability to accommodate volume (Table 10). For all cases except DFW 2006, which is under capacity for the entire day, the number of diverted flights for the static radius exceeded the number for the dynamic radius.

\begin{tabular}{|c|c|c|c|}
\hline Airport & $\begin{array}{c}\text { Traffic } \\
\text { Set }\end{array}$ & $\begin{array}{c}\text { Radius } \\
\text { Mode }\end{array}$ & $\begin{array}{c}\text { Number } \\
\text { Diverted }\end{array}$ \\
\hline ATL & 2018 & static & 347 \\
\hline ATL & 2018 & dynamic & 13 \\
\hline ATL & 2006 & static & 92 \\
\hline ATL & 2006 & dynamic & 2 \\
\hline DFW & 2018 & static & 95 \\
\hline DFW & 2018 & dynamic & 35 \\
\hline DFW & 2006 & static & 6 \\
\hline DFW & 2006 & dynamic & 6 \\
\hline \multicolumn{4}{|c}{ Table 10. Diverted Flights } \\
\end{tabular}




\section{Summary}

The concept system used a dynamic terminal airspace that expanded the arrival planning radius from the approximate size of a current day TRACON (50 nmi) to as large as $130 \mathrm{nmi}$ when necessary to accommodate predicted arrival traffic volume. The system was designed to improve arrival capacity of the airport when traffic volume demanded. ACES with M\&S was used to host and test the concept system. Four scenarios using a combination of ATL and DFW with 2018 and 2006 traffic sets were used to test arrival scenarios ranging from under to over-capacity with current day STAR routes and airport configuration.

Results showed significant throughput increase for scenarios that are considered to be over-capacity for current day. During periods of sustained demand for the ATL 2018 case, throughput increased by 26 operations per hour $(30 \%)$ and average delay was reduced from 18 minutes to 8 minutes per flight when using the TACS system with a dynamic planning radius. Similar results were obtained for DFW with 2018 traffic levels and for ATL with 2006 traffic levels, but with lower benefits due to lower demand. For the DFW 2006 scenario, the results with and without the TACS system were identical because under-capacity arrival traffic never necessitated the radius to increase.

The concept system, TACS, was also tested to verify that two different configuration objectives (minimized runways or minimized radius) provided the same throughput regardless of the objective selected. A nearly identical time history resulted, and outputs for the percentage of time in 1,2 , and 3 runways and the percentage of time in radii were included to demonstrate the functionality of the option. This option would offer benefit to airports that are not over-capacity, but wish to consolidate arrival traffic to fewer runways to potentially accommodate increased departure runway space.

\section{References}

[1] Glaab, Patricia, 2012, Improvement to Airport Throughput Using Intelligent Arrival Scheduling and an Expanded Arrival Planning Horizon, NASA/TM-2012-217762.
[2] Traffic Flow Management in the National Airspace System, 2009, http://www.fly.faa.gov/Products/Training/Traffic Management for Pilots/TFM in the NAS Book1 et_ca10.pdf

[3] Airspace Concepts Evaluation System, NASA Aviation System Division, May 5, 2009, http://www.aviationsystemsdivision.arc.nasa.gov/re search/modeling/aces.shtml

[4] Santos, Michel, et al, Aug. 2-5, 2010, Scheduling the Use of Airborne Merging and Spacing Along Multiple Converging Routes to an Airport, Toronto, Ontario, AIAA Modeling and Simulation Technologies Conference, 2010-8366.

[5] Barmore, Bryan, Terence Abbott, Karthik Krishnamurthy, Airborne-Managed Spacing in Multiple Arrival Streams, 2004, 24 ${ }^{\text {th }}$ International Congress of the Aeronautical Sciences.

[6] EUROCONTROL, European Organisation for the Safety of Air Navigation, April 2013, Base of Aircraft Data (BADA), http://www.eurocontrol.int/services/bada.

[7] Viken, Jeffrey, et al, September 2006, Utilizing Traveler Demand Modeling to Predict Future Commercial Flight Schedules in the NAS, Portsmouth, Virginia, $11^{\text {th }}$ AIAA/ISSMO Multidisciplinary Analysis and Optimization Conference, 2006-7032.

[8] Capacity Needs in the National Airspace System 2007 - 2025 (FACT 2), May 2007, The MITRE Corporation, FAA Publication HQ009107.indd, http://www.faa.gov/airports/resources/publications/ reports/media/fact_2.pdf

\section{Email}

patricia.c.glaab@nasa.gov

\author{
32nd Digital Avionics Systems Conference \\ October 6-10, 2013
}

